\title{
A Syndrome of Mental and Physical Retardation, Speech Disorders, and Peculiar Facies in Two Sisters
}

\author{
OSVALDO MUTCHINICK
}

\author{
From the Medical Genetics Center, Combate de los Pozos 2193, Buenos Aires, Argentina
}

Two sisters, born to consanguineous parents, presumed to be affected with a new syndrome of mental retardation and multiple congenital anomalies are reported.

\section{Case Reports}

Case 1 (IV.1) (see Fig. 1). This girl was born on 21 September 1961. Her mother was 22 and her father 24 years old at the time of birth. The only known unusual event during pregnancy was a large umbilical hernia and an abdominal $x$-ray was taken at about 30 weeks of gestation. Labour was induced by amniotomy and oxygen was given during labour because of a tetanic contraction. Delivery was uneventful but the newborn was anoxic and required active resuscitation. The birth weight is unknown. She remained in the nursery for a week because of feeding difficulties and occasional vomiting of blood.

Both physical and motor developments were slow from birth and during the first 3 months of life she had frequent crises of irritability and hypertonicity. She first raised her head at 4 months, sat at 6 months, and started walking at 30 months. At 18 months she began to speak isolated words and was able to construct short sentences at 48 months; there was then evidence of a speech impairment which persists to the present time. She had had no other serious illness.

Physical examination. She was first examined by the author at $7 \frac{1}{2}$ years. The findings at that time were as follows: weight $17.5 \mathrm{~kg}$; height $110 \mathrm{~cm}$; vertex-pubis $57 \mathrm{~cm}$; head circumference $45 \mathrm{~cm}$; chest circumference $57 \mathrm{~cm}$. All these measurements, except for the chest circumference, were below the 3 rd centile for age and sex.

The significant positive physical findings were: microbrachycephaly with narrow sloping forehead; marked hypertelorism, small palpebral fissures (blepharophimosis) with anti-mongoloid slant; long curly eyelashes, light blue iris, severe photophobia, hypermetropia, and normal fundi. The nose was broad and straight with a lack of depression at the bridge. The mouth was wide-tented, with outward and downward slant of the corners, the upper lip covering the visible mucosal portion of the lower lip. She had a high

Received 17 May 1971. arched palate and dental anomalies such as malocclu- $-\frac{\text { S }}{3}$ sion, misplaced teeth slanting inwards at $45^{\circ}$, and irregular edges of the incissors. There was moderate 9 prognathism. Her ears were very large and flared with $\overrightarrow{-}$ a lack of differentiation of the antihelix branches. Her 8 chest was pigeon shaped with anterior sternocostal pro- 윽 trusion. A systolic murmur was heard best over the pulmonic area and the second heart sound was split. $\bar{z}$ Scanty, thin, and blonde pubic hair was present. Her skin was white, with 15 moles, none larger than $2 \mathrm{~mm}$ in $\stackrel{\rho}{)}$ diameter, distributed on the chest and abdomen. Hair $\vec{\theta}$ was thin, blonde, rather scarce, and normally implanted. She had hyperconvex thumb nails and bilateral clino- ? dactyly of the 5th finger. She had severe mental 0 retardation $(I Q=42)$ with speech disorders: dyslalia, anomia, and agrammatism. She also had a broad standing base and a spastic gait.

The following studies were within normal limits: haemogram, serum calcium, phosphate, uric acid, protein-bound iodine, carbohydrate and protein electrophoresis, and lipidogram. Studies of urine calcium, carbohydrate, amino-acid chromatography, and 17ketosteroids were also normal. The qualitative urinary tests for phenylpyruvic acid, ketoacids, cysteine, homocysteine, tyrosine, hydroxyphenylpyruvic acid, and mucopolysacharides were all negative. The only abnormal result was an increased 17-ketogenico-steroid excretion of $12.6 \mathrm{mg}$ in 24 hours.

Radiological findings. The skull $x$-ray showed thin frontal diploe, small posterior fossae, orbital hyperteleorism, and low posterior part of the mandibular horizontal branch. The chest $x$-ray showed premature sternal fusion, sternocostal forward protrusion, and cuboid shaped dorsolumbar vertebral bodies. $X$-ray of the pelvis showed open ischiopubic synchondrosis. The $x$-ray of the limbs showed a small carpus and normal bone age; generalized osteoporosis was present. An IVP showed a moderate bilateral calyceal dilation with dilated right ureter; the left ureter could not be seen well. ECG demonstrated a complete right bundle branch block and findings compatible with interauricular septal defect plus valvular pulmonic stenosis. EEG showed a defective central bioelectric organization.

Case 2 (IV.3) (see Fig. 1). This girl was born on 12 June 1965 after an uncomplicated pregnancy of 40 weeks. She was exposed to $x$-rays during the 5 th 

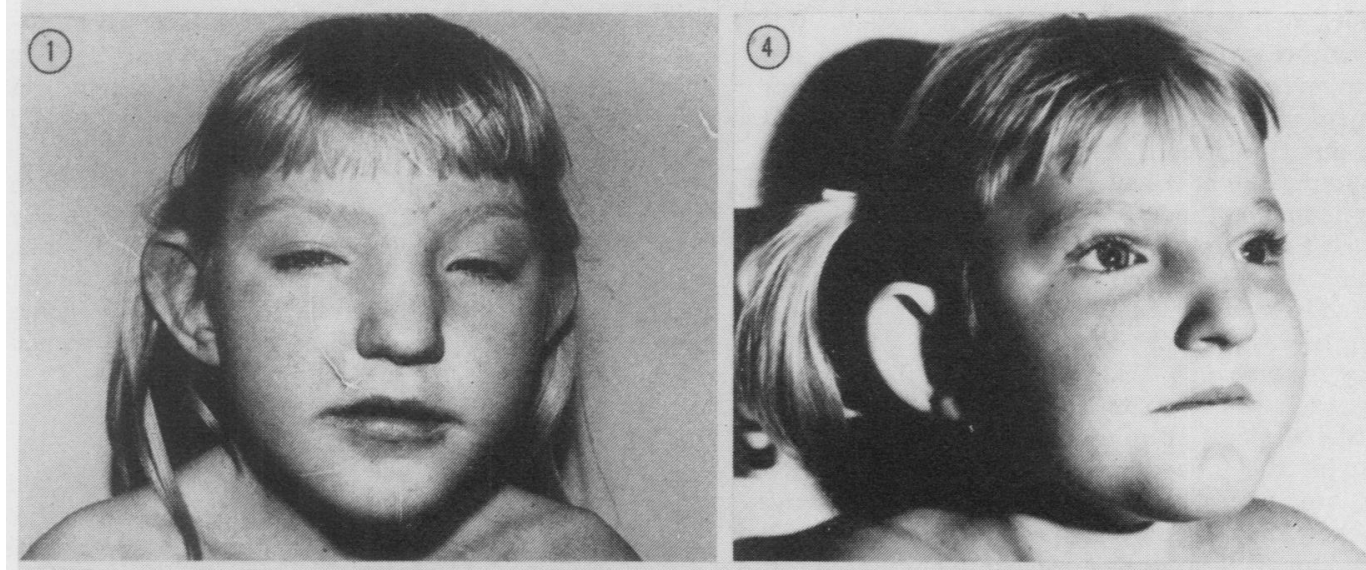

(2)
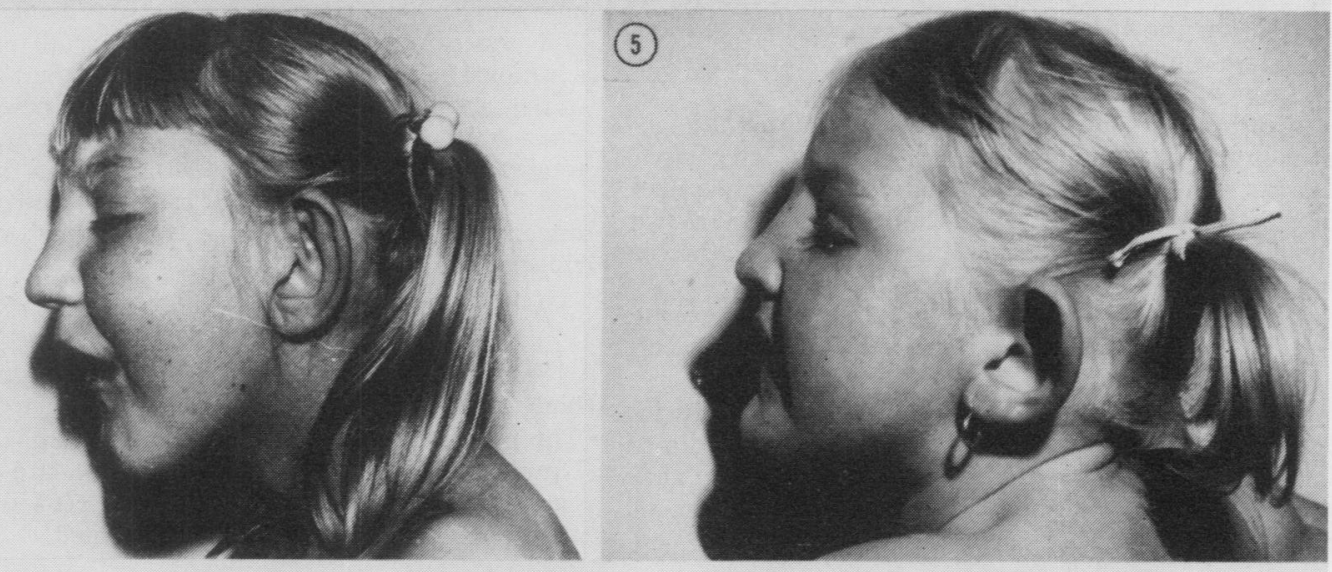

(3)
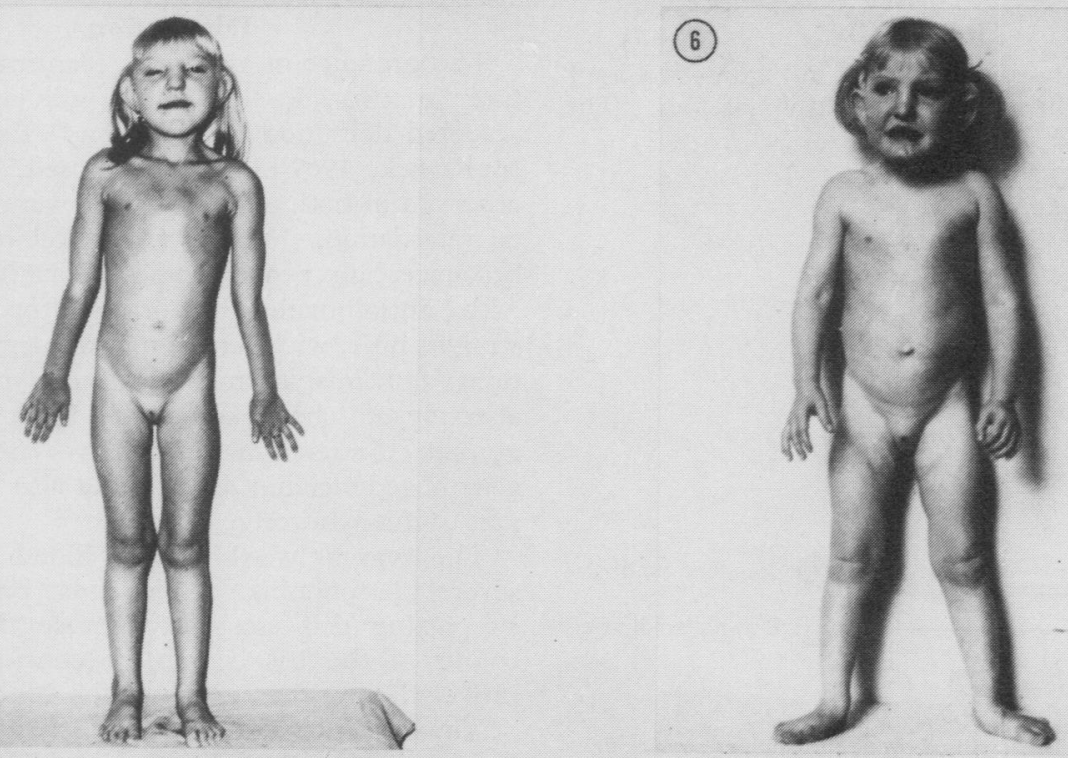

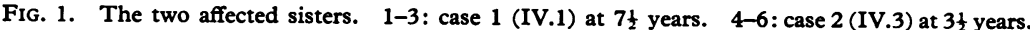


month of fetal life while case 1 was being examined by $x$-ray. Her delivery was normal; birth weight was $3800 \mathrm{~g}$. Although the neonatal period was normal and there were no feeding difficulties her physical and motor development was markedly slow from early infancy; this pattern being similar to that described in case 1 . The patient had measles at the age of 3 years, with secondary bronchopneumonia and unconsciousness; the spinal fluid at that time was normal. No changes in her clinical picture were observed after this episode. The patient died from an accidental burn at the age of 4 years. No necropsy was performed.

Physical examination. This patient's physical appearance was strikingly similar to that of her affected sister. She was first examined at the age of 3 years 10 months with the following findings: weight $13.5 \mathrm{~kg}$; height $92 \mathrm{~cm}$; vertex-pubis $50 \mathrm{~cm}$; head circumference $44 \mathrm{~cm}$; chest circumference $53.5 \mathrm{~cm}$. Like her sister all measurements, except for the chest circumference, were below the 3rd centile for age and sex. Because of her close similarity to her sister, only the discordant findings will be given.

Findings present only in case 2. Bilateral genuvalgum and pes-valgum, rotated right kidney, left calyceal dilatation, and radiological amputation of all renal papillae. The ECG was normal, even though an interauricular septal defect was diagnosed clinically. The megacolon was suspected on radiological grounds.

Findings present only in case 1. Pubic hair present, bilateral blepharophimosis, right bundle branch block, and valvular pulmonic stenosis.

Laboratory analyses (haemogram, serum and urine carbohydrate, and protein electrophoresis), performed in case 2 , were all within normal limits. The same qualitative urinary tests described for case 1 were all negative.

\section{The Family}

An abbreviated pedigree is given in Figure 2. Case 1 (IV.1) was the first born in a sibship of four. The

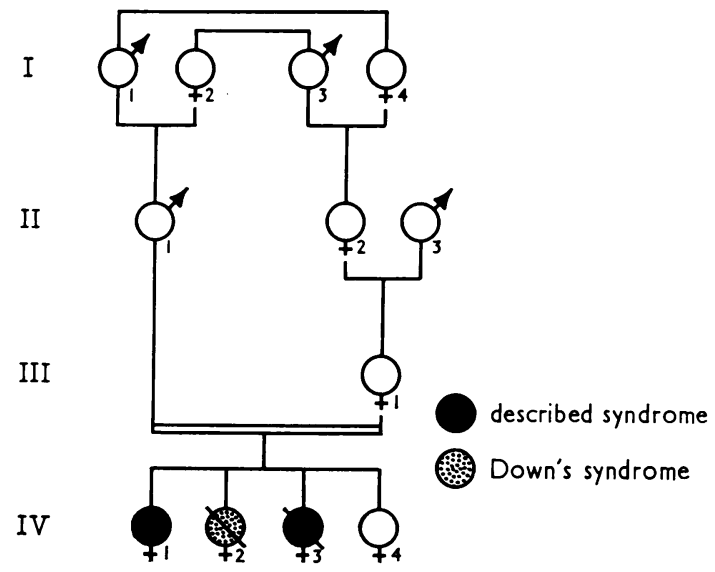

FIG. 2. Pedigree of the family showing the complex consanguinity of the parents (II.1 and III.1) of the affected girls (IV.1 and IV.3). second born (IV.2) was a girl said to have had Down's syndrome who died at the age of 2 months from an un- $\mathbb{D}$ known cause. The third sib (IV.3) was case 2 . The $\underset{\oplus}{\oplus}$ fourth child, another girl (IV.4) who was 5 months old at the time of this report, was examined by the author and $\stackrel{5}{?}$ no abnormal signs were observed.

The parents are double cousins once removed. Both $\frac{\overline{0}}{0}$ the father's and mother's ancestors come originally $\frac{\bar{c}}{2}$ from neighbouring villages of 300 to 500 inhabitants, $\overparen{\varnothing}$ situated in the area of the old Russio-Germanic frontier 2 (1914). It is interesting that one of the family branches of is called Kölln while one of those two villages is named $\vec{\circ}$ Köln.

\section{Dermatoglyphs}

Dermatoglyphic patterns were analysed in the affected sisters, the normal sister (IV.4), and the parents. The father's left 3rd finger was missing because of accidental amputation. Radial loops were only present in the left 8 2nd fingers; in the affected girls and both parents, an 을 additional radial loop was present in the right 2 nd finger of the mother and in the left 4th finger of case 1 . Only $\bar{z}$ cases 1 and 2 had vestigial loops on thenar areas. The total ridge count was 73 in the father, 100 in the mother, 宁 and 119 and 116 in case 1 and case 2 respectively. The fingerprints of IV.4 were not clear enough to allow a ridge count to be made.

\section{Cytogentic Studies}

Sex chromatin determination on buccal smears from both patients and parents were according to their sex. ̊ँ Chromosome studies on cultured peripheral blood $\mathbb{\AA}$ lymphocytes were performed in cases 1 and 2 and the $\vec{F}$ parents and special care was taken to detect any structural anomalies; no abnormalities were detected.

\section{Discussion}

The presence of an almost identical clinical picture in two closely-related individuals fits the accepted definition of 'syndrome' (Edwards, 1969; McKusick, 1969). The diagnosed cardinal signs observed in both affected sisters were: severe mental retardation, slow physical development, microbrachycephaly, peculiar facies (ocular hyperteleorism with antimongoloid slant, photophobia, broad straight nose, wide tented mouth, dental malformations and misplacements, large dysplastic ears); o also pigeon chest, congenital heart disease, and $N$ non-specific renal malformations. Some degree of $\mathrm{W}$ generalized melanin defect could also be considered part of this syndrome.

The very light white skin, blond hair, and the severe photophobia are the most important signs supporting this suggestion, since the light blue colour of the iris cannot be considered as definitively lighter than expected for this family.

The existence of a mongoloid sib and some facial features of the affected girls raised the possibility of 


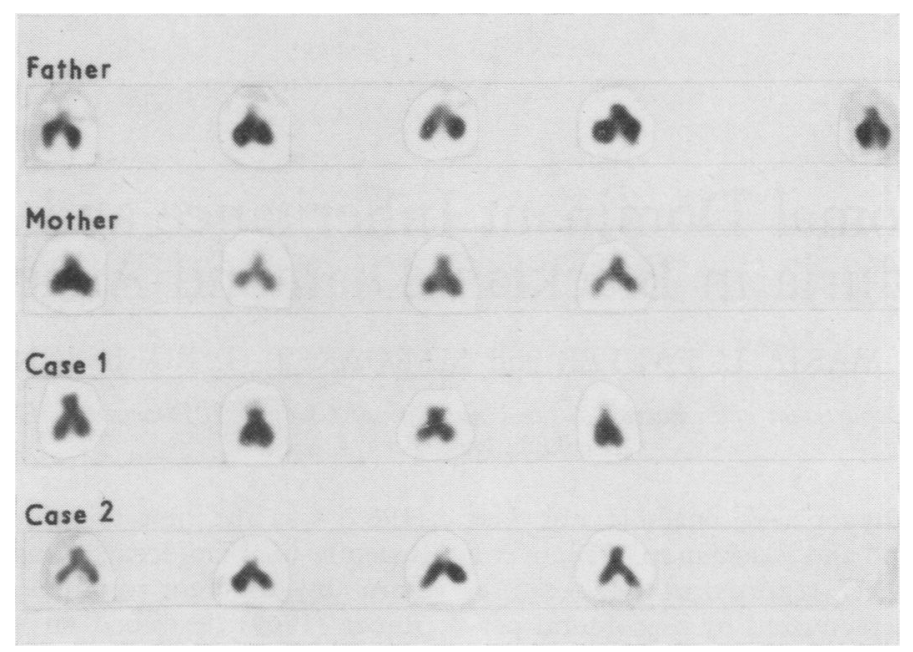

FIG. 3. G group chromosomes of the parents and the affected daughters.

a balanced translocation in one of the parents involving a chromosome of the $G$ group, with a partial trisomy in the mongoloid girl and a deletion of a $\mathrm{G}$ chromosome in the two affected sisters.

No chromosomal abnormalities, however, were detected, even though submicroscopic structural changes could not be ruled out. Fig. 3 shows the G group chromosomes of the individuals examined.

The clinical picture of this condition was compared with several recognized syndromes with microcephaly, and mental retardation (Gellis and Feingold, 1968; McKusick, 1968; Goodman and Gorlin, 1970; Smith, 1970). The new syndrome proposed here shared some features with true recessive microcephaly and Seckel's bird-headed dwarfism such as: microcephaly, mental retardation, and small stature. It also had some additional signs of recessive microcephaly (sloping forehead, high arched palate, large and protruding ears) and of Seckel's dwarfism (renal disorders, dental anomalies, sparse hair, clinodactyly), but the whole appearance of the face, prognathism, severe photophobia, pigeon chest, and cardiac and renal malformations are evidence of a different entity.

The observed recurrence of this syndrome in two sisters born to healthy consanguineous parents, and the close geographical origin of their ancestors strongly suggest a simple Mendelian autosomal recessive type of inheritance for this syndrome in this family.
The unsuccessful search for a similar report in the medical literature leads this author to propose these two cases as examples of a new syndrome.

\section{Summary}

A family is described with two sisters affected by a previously undescribed syndrome of mental and physical retardation, speech disorders, and peculiar facies due to ocular hyperteleorism, antimongoloid slant, photophobia, broad straight nose, wide tented mouth, dental anomalies, and large dysplastic ears, with other congenital anomalies. The parents were healthy and consanguineous. An autosomal recessive inheritance is proposed for this syndrome.

\section{REFERENCES}

Edwards, J. L. (1969). Summing up the conference. Birth Defects: Original Article Series, V, 2. The National FoundationMarch of Dimes, New York.

Gellis, S. S. and Feingold, M. (1968). Atlas of Mental Retardation Syndromes. US Department of Health, Education and Welfare, US Government Printing Office, Washington DC.

Goodman, R. M. and Gorlin, R. J. (1970). The Face in Genetic Disorders. C. V. Mosby, San Louis.

McKusick, V. A. (1968). Mendelian Inheritance in Man, 2nd ed. Johns Hopkins Press, Baltimore.

McKusick, V. A. (1969). On lumpers and spliters, of the nosology of genetic disease. Birth Defects: Original Article Series, V, 1. National Foundation-March of Dimes, New York.

Smith, D. W. (1970). Recognizable Patterns of Human Malformations. (Major Problems in Clinical Pediatrics, vol. 7.) Saunders, Philadelphia. 